\title{
Pulse laser surface modification of poly-ether-ether-ketone for surface metallization
}

\author{
Nan Wang ${ }^{1 *}$, Jia-feng $\mathrm{Li}^{1}$, Jing-ying Bai ${ }^{1}$, Xu-guang Wang ${ }^{1}$ and Li-gong Zhang ${ }^{1}$ \\ ${ }^{1}$ China Academy of Space Technology, Beijing Spacecrafts, Beijing (China), 100094
}

\begin{abstract}
In order to broaden the application range, PEEK has been modified by $1064 \mathrm{~nm}$-wavelength pulse laser, then deposited with Ni-P alloys by electroless plating. The properties of metal layers on PEEK were characterized by SEM, resistance tester and metallographic microscope, respectively. When the laser energy density is relatively low $\left(\mathrm{Q}<60 \mathrm{~J} \cdot \mathrm{cm}^{-2}\right)$, there is mainly photothermal effect on the PEEK with laser, and the surface of the substrate presents periodic undulating groove structure with a small number of holes. When the laser energy density is rather higher $\left(\mathrm{Q} \geq 60 \mathrm{~J} \cdot \mathrm{cm}^{-2}\right)$, there are simultaneously enhanced photothermal effect and partial photochemical effect. As a result, the surface of PEEK is uniformly covered with melted layer or re-solidified product after melting. Besides, the nickel coatings on the PEEK have been dense, uniform and in good adhesion with the substrate for the sample after laser treatments $\left(\mathrm{Q}>10 \mathrm{~J} \cdot \mathrm{cm}^{-2}\right)$, of which the surface resistance of the coating is less than $20 \mathrm{~m} \Omega$. In this paper, the interaction mechanism of the pulse laser on PEEK has been studied and the metals on the PEEK have been prepared under the laser-induced effect, which provides technical support for the future application of PEEK in aviation with aerospace fields, such as, radar and antenna.
\end{abstract}

\section{Introduction}

Poly-ether-ether-ketone (PEEK) is a kind of semi-crystalline aromatic thermoplastics with outstanding features, such as excellent mechanical properties [1,2] (young's modulus, $4 \mathrm{GPa}$; tensile strength, $150 \mathrm{MPa}$ ), thermal stability $[3,4]$ (glass transition temperature, $143^{\circ} \mathrm{C}$; melting point $343^{\circ} \mathrm{C}$; decomposition temperature, $580{ }^{\circ} \mathrm{C}$ ), radiation resistance $[5,6](1100 \mathrm{M} \gamma$ radiation without significant degradation) and chemical corrosion resistance [7-10], which make it attractive to the fields of medical and health, as well as aerospace [11-16]. However, it is usually necessary to have superior conductivity on the surface to meet the electrical properties of the products when they are applied, which is contrary to the high insulation properties of the polymers, such as PEEK. Therefore, it is an implementable method of continuous metal layers on the surface of PEEK with the high stability, which gives the product good conductivity and electromagnetic shielding performance, to satisfy the need of its application in electronic circuit, radar transmitter and so on.

As is known, PEEK materials have smooth and dense surface, high chemical inertness, only dissolved in high concentration sulfuric acid, and the metal layer prepared on the surface of reaction products has poor adhesion. Therefore, the pretreatment with the surface of the substrate is a key factor that affects the bonding strength of the metal layer on the PEEK.

Compared with the traditional chemical modification, physical modification and other technologies of surface modification, the pulsed laser for surface modification has the advantage of no mask, no pollution, high accuracy and fast processing speed [17,18], in particular, it can change selectively the physical and chemical properties of the substrate. This surface modification with the laser has been applied to the metallization pretreatment of polycarbonate, polyurethane and other plastic surfaces [19-21], therein, the micromorphology and chemical composition of the material are analyzed under the action of the pulse laser with different frequency, different energy density and spot overlap ratio. It is found that the influence mechanism of pulse ultraviolet picosecond/nanosecond laser and pulse infrared picosecond/nanosecond laser on the surface of engineering plastics is different. The ultraviolet laser mainly produces photochemical reaction with the substrate, whereas infrared laser mainly produces photothermal reaction. The photothermal effect and/or photochemical effect due to the pulse laser at the interface of materials would make the interface region melted in a very short time, which produces the structure of pit, groove, and melt accumulation, meanwhile, the different contents of $\mathrm{C}, \mathrm{N}$ and $\mathrm{O}$. Inspired by this, it can be considered that laser treatment would modify the surface morphology and composition of PEEK materials, but there is no relevant research on the surface laser modification of PEEK materials, especially the influence of laser treatment on the deposition effect of metal layer on the modified PEEK.

In this paper, the surface of PEEK was modified by 
$1064 \mathrm{~nm}$ pulse infrared laser. Then the influence of laser energy density on the surface morphology of PEEK was studied, and the mechanism of photothermal and/or photochemical interaction between the laser and PEEK was explored. After that, the metal layer with continuous and dense nickel was deposited on the surface of PEEK modified by the laser, where the effect of laser treatment on the adhesion of metal layer was analyzed. Pulse laser modified PEEK for surface metal layer deposition is simple and has good local selectivity. The deposited coating has good conductivity, compact structure and high adhesion with the substrate, which can achieve good surface conductivity and electromagnetic shielding performance of PEEK products, as a result, lay a technical foundation for its future application in aerospace and other fields.

\section{Experimental Section}

\subsection{Materials and Equipments}

PEEK (Nanjing Julong Science \& Technology Co., LTD, $40 * 40 * 2 \mathrm{~mm})$.

The needed solvents were activating solutions $\left(\mathrm{PdCl}_{2}\right.$ $0.5 \mathrm{~g} / \mathrm{L}$, hydrochloric acid $5 \mathrm{~mL} / \mathrm{L}$ ) and the reducing solution (Sodium hypophosphite 20 40 g/L), as well as the electroless nickel-plating solution, of which the specific composition is shown in Table 1.

The main equipment used in the preparation was $1064 \mathrm{~nm}$-wavelength infrared pulse fiber laser (Shenzhen Laser-MID 3D-Circurt Technology Co., Ltd). The maximum output power is about $30 \mathrm{~W}$, the diameter of focusing spot is about $20 \mu \mathrm{m}$, the repetition frequency of the laser is adjustable in the range of $10 \sim 100 \mathrm{kHz}$, and the corresponding pulse breadth is $10 \sim 60 \mathrm{~ns}$.

Table 1. The specific composition of the electroless nickel-plating solution.

\begin{tabular}{ccc}
\hline Content & Molecular formula & Concentration \\
\hline Nickel sulfate & $\mathrm{NiSO}_{4} \cdot 6 \mathrm{H}_{2} \mathrm{O}$ & $20 \sim 30 \mathrm{~g} / \mathrm{L}$ \\
Sodium hypophosphite & $\mathrm{NaH}_{2} \mathrm{PO}_{2}$ & $10 \sim 20 \mathrm{~g} / \mathrm{L}$ \\
Sodium pyrophosphate & $\mathrm{Na}_{4} \mathrm{P}_{2} \mathrm{O}_{7} \cdot 10 \mathrm{H}_{2} \mathrm{O}$ & $100 \sim 130 \mathrm{~g} / \mathrm{L}$ \\
Sodium succinate & $\mathrm{C}_{4} \mathrm{H}_{4} \mathrm{O}_{4} \mathrm{Na}_{2}$ & $10 \sim 20 \mathrm{~g} / \mathrm{L}$ \\
Ammonium bifluoride & $\mathrm{NH}_{4} \mathrm{HF}_{2}$ & $5 \sim 15 \mathrm{~g} / \mathrm{L}$ \\
Saccharin sodium & $\mathrm{C}_{6} \mathrm{H}_{4} \mathrm{SO}_{2} \mathrm{NNaCO}_{2} \cdot 2 \mathrm{H}_{2} \mathrm{O}$ & $1 \sim 3 \mathrm{~g} / \mathrm{L}$ \\
Sodium dodecyl sulfonate & $\mathrm{C}_{12} \mathrm{H}_{25} \mathrm{O}_{3} \mathrm{Na}$ & $0.01 \sim 0.1 \mathrm{~g} / \mathrm{L}$ \\
Aqueous ammonia & $\mathrm{NH}_{3} \cdot \mathrm{H}_{2} \mathrm{O}$ & $20 \sim 40 \mathrm{~mL} / \mathrm{L}$ \\
Stabilizer & $/$ & $14.9 \mathrm{~mL} / \mathrm{L}$ \\
\hline
\end{tabular}

\subsection{Methods}

Degreasing $\longrightarrow \begin{gathered}\text { Laser } \\ \text { corrosion }\end{gathered} \longrightarrow$ Rinsing $\longrightarrow \begin{gathered}\text { Surface } \\ \text { activation }\end{gathered} \longrightarrow$ Rinsing $\begin{gathered}\text { Chemical } \\ \text { nickel-plating }\end{gathered}$

Fig. 1. Test on the surface metallization of PEEK assisted by laser.

At first, the PEEK materials were placed in alcohol solution for about $15 \mathrm{~min}$ to remove the oil stain on the surface of the samples, followed by rinsing with deionized water (Fig. 1). Then the samples were carried out with laser using $1064 \mathrm{~nm}$-wavelength infrared (IR) pulse fiber laser, of which the treatments were performed in air at atmospheric pressure. In this process, to investigate the effect of laser on the surface properties of the PEEK, the samples with increased laser energy densities $\left(\mathrm{Q}=0 \sim 180 \mathrm{~J} \cdot \mathrm{cm}^{-2}\right)$ were obtained through altering the laser power, pulse frequency, scanning rate and spot overlap ratio (Table 2).

Subsequently, the laser treated samples understood surface activation, processed with activating solutions (palladium chloride solution) and the reducing solution (Sodium hypophosphite), before and after rinsing with water. Soon afterwards, the surface activated samples were put into electroless nickel-plating solution at $78 \sim 80{ }^{\circ} \mathrm{C}$ for $30 \sim 40 \mathrm{~min}$, aimed to the process of Ni-P alloy deposition.

Table 2. Experimental design of etching PEEK with pulsed Infrared laser

\begin{tabular}{ccccccc}
\hline $\begin{array}{c}\text { Frequency } \\
{[\mathbf{k H z}]}\end{array}$ & $\begin{array}{c}\text { Power } \\
{[\mathbf{W}]}\end{array}$ & $\begin{array}{c}\text { Scan rate } \\
{\left[\mathbf{m m ~ s}^{-1}\right]}\end{array}$ & $\begin{array}{c}\text { Scan space } \\
{[\boldsymbol{\mu m}]}\end{array}$ & $\begin{array}{c}\text { Crosswise } \\
\text { overlap ratio } \\
{[\%]}\end{array}$ & $\begin{array}{c}\text { Lengthwise } \\
\text { overlap ratio } \\
{[\%]}\end{array}$ & $\begin{array}{c}\text { Energy density } \\
{\left[\mathbf{J} \cdot \mathbf{c m}^{-2}\right]}\end{array}$ \\
\hline $10 \sim 100$ & $3 \sim 30$ & $500 \sim 2000$ & $10 \sim 120$ & -25 & -100 & $0 \sim 180$ \\
\hline
\end{tabular}

\subsection{Characterization}

The samples were inspected making use of scanning electron microscopy (SEM, JEOL, JSM-6701F) and metallurgical microscope in frontal view to the laser treated area.
The surface resistance of the metallized sample was measured by resistance tester.

According to GB/T 5270-2005, the grid test was carried out to verify the adhesion of the metal coating on the surface of the specimen.

According to GJB 150.5A-2009, the hot and cold 
impact test was carried out at $-196{ }^{\circ} \mathrm{C} \sim 100{ }^{\circ} \mathrm{C}$ for 10 cycles, and the adhesion state of the coating surface was observed for blistering, peeling or delamination, so as to verify the test performance of the metal coating on the surface of the specimen under high and low temperature harsh environment.

\section{Results and discussion}

\subsection{Surface micromorphology of PEEK with laser modification at different energy density}

In the field of surface engineering, in order to realize the special functional properties of the material surface, in addition to the properties of the material itself, the three-dimensional micro-structure characteristics at the material interface also have a very important relationship with its macro-properties, for example, the wettability, adsorption, corrosion resistance of the material surface, and the bonding strength of the metal layer on the substrate surface. Therefore, by laser surface modification of PEEK, different micro-characteristics can be obtained at the material interface, which not only affects the morphology and composition of PEEK substrate, but also has a great influence on the bonding strength of the deposited metal layer. Fig. 2 is the surface of the etched PEEK with laser energy density under the action of pulsed laser at a fixed overlap ratio of the laser spot $\left(\varphi_{\mathrm{x}}=-25 \%, \varphi_{\mathrm{y}}=-100 \%\right)$. The original PEEK presents, with the increase of pulse laser energy density, the color of PEEK surface, light tea gray, becomes deepens gradually after laser treatment, and finally appears gray black, which indicates that pulse laser changes the micro-morphology of PEEK, so that the macro reflection of light changes, and thus presents different color states.

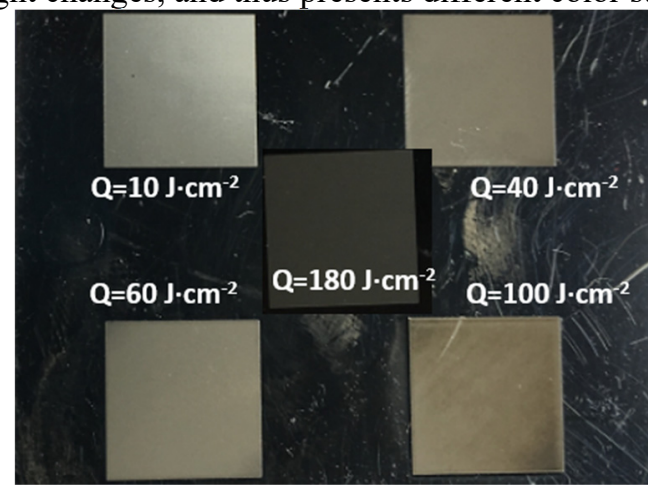

Fig. 2. Images of PEEK surface after laser treatments.

The surface morphology of PEEK modified by laser with different energy density was characterized, as shown in Fig. 3. It can be seen that the PEEK without laser treatment presents the regular rough surface after injection processing, and the microstructure presents a relatively uniform uneven state, and the uneven degree is relatively flat. Due to the hydrophobicity of PEEK substrate, the plating solution can not enter into the concave area of micro-interface, which is not conducive to the deposition of metal layer. When pulsed laser is used to modify the surface of organic polymer, the lower energy density will be absorbed, reflected and scattered by the substrate interface. Only when the energy density of pulsed laser irradiating the substrate reaches a certain degree, the physical and chemical comprehensive reaction effect at the substrate interface will be caused, and changed the micro-morphology of the material surface significantly.
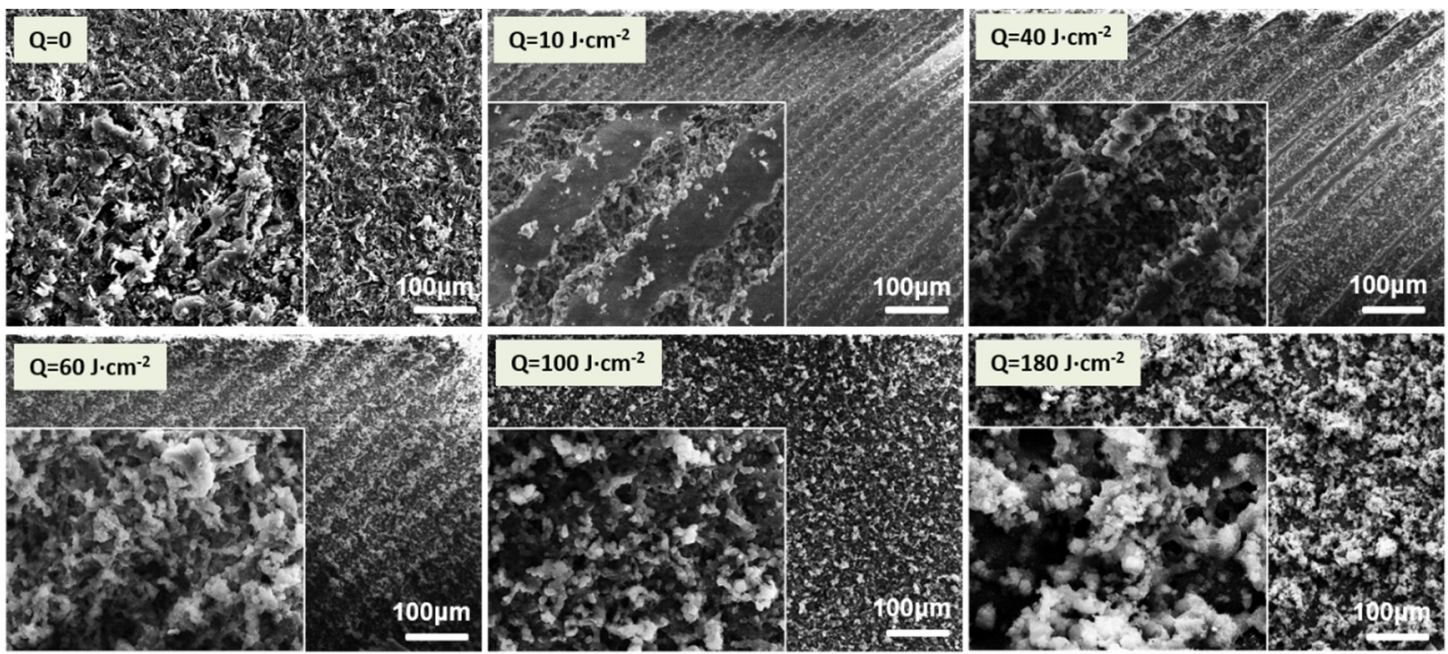

Fig. 3. SEM of PEEK surface after laser treatments.

From the SEM results of PEEK after pulsed laser treatment, when the laser energy density reaches to $Q=10$ $\mathrm{J} \cdot \mathrm{cm}^{-2}$, the surface of PEEK shows obvious etching morphology. However, due to the relatively low energy density, when the laser reaches the interface of PEEK substrate, the laser energy is basically absorbed and scattered, resulting in the melting and vaporization of the organic polymer in the treated area, and the substrate in some areas is melted and cooled after vaporization, which makes the micro-area of the material surface appear relatively regular morphological characteristics.

In addition, there is a relatively obvious boundary between the etched and the non-etched surface at the interface of PEEK substrate irradiated by low energy 
density laser.

The micro-roughness of the surface with direct irradiation is rather rougher, while the non-irradiation area presents a relatively smooth surface state. This may be due to the material damage in the very shallow surface area at the interface of PEEK substrate in the non-irradiation surface when the laser energy density is low. Whereas, due to the low energy, gasification effect is not produced, and then after rapid cooling, it presents a relatively smooth surface state. The energy density of the laser direct irradiation area is relatively high, and the organic polymer melting and gasification occurs in the very shallow area of the substrate interface, resulting in the rough effect. With the increase of laser energy density, the surface of PEEK is slightly damaged by pulse laser. The results show that the induced etching effect of the apparent state increases gradually, and the influenced surface of the substrate also extends and expands gradually, so that, the PEEK at the interface not only melts and vaporizes in the longitudinal direction, but also in the transverse direction, as a result, the whole surface area of the substrate presents a periodic micro three-dimensional undulating structure $\left(\mathrm{Q}=40 \mathrm{~J} \cdot \mathrm{cm}^{-2}\right)$.

At the same time, it can be seen from Fig.3 that under the condition of the laser energy density at $40 \mathrm{~J} \cdot \mathrm{cm}^{-2}$, there are still relatively regular protrusions at the boundary, indicating that there are still relatively weak spots on the surface of the whole peek substrate, which make in failure the interface region of the substrate produce relatively uniform three-dimensional structure. With the continuous increase of laser energy density $\left(\mathrm{Q}=60 \mathrm{~J} \cdot \mathrm{cm}^{-2}\right.$ and $\left.\mathrm{Q}=100 \mathrm{~J} \cdot \mathrm{cm}^{-2}\right)$, the amount of melt on the peek surface increased gradually after laser etching, and the periodic undulating boundary became blurred gradually. Finally, the obvious morphology of melt accumulation appeared.

Besides, it can be observed that when the laser energy density reaches $180 \mathrm{~J} \cdot \mathrm{cm}^{-2}$ (Fig. 3), the micro periodic fluctuation structure of PEEK surface after pulse laser treatment is accompanied by a small number of micro holes, which gradually increase with the laser energy density increased. This is because when the laser energy density is high, not only the melting and gasification of organic polymer occurs in the very shallow surface of the PEEK, but also the secondary dissolution and gasification effect occurs, showing more micro pores.

\subsection{Interaction between pulsed infrared laser and PEEK}

The PEEK shows variable morphology after etching with different energy density, which is related to the interaction mechanism between pulse laser and organic polymer materials. The interaction between pulse laser and polymer is based on photon energy. As known, the way of absorbing pulse laser photons on the material surface is affected by the laser energy density. When the pulse laser energy density is low, single photon absorption takes place mainly on the PEEK, while when the laser energy density is large, there is mainly multi-photon absorption. When the pulse laser irradiates the surface, due to the difference of photon energy and chemical bond energy in the material molecule, there is photochemical reaction or photothermal reaction occurred, which affects the micro-morphology of PEEK surface after laser etching [22].

The single photon energy $(1.16 \mathrm{eV})$ of $1064 \mathrm{~nm}$ pulsed fiber laser is obviously less than that of all chemical bonds in PEEK molecule (as shown in Table 3). Therefore, when the energy density of pulse laser is low, the photothermal effect is the main one when the laser acts on the PEEK (as shown in Fig. 4). The transient thermal energy causes thermal expansion, melting and gasification effects in the very shallow area of PEEK substrate interface, and the thermal expansion, melting and gasification effects occur in the very shallow area. When the laser energy density increases to a certain extent, the single and multi-photon absorption occur simultaneously when the laser irradiates the material surface, which makes the photothermal effect on the PEEK more obvious, and the surface is melted or re-condensed after melting. The number of solid organic chain breaking polymers increased significantly, and gradually connected into pieces and accumulated $(\mathrm{Q}=60$ $\mathrm{J} \cdot \mathrm{cm}^{-2}$ and $\mathrm{Q}=100 \mathrm{~J} \cdot \mathrm{cm}^{-2}$ ).

Table 3. Bond energy of chemical bond in PEEK materials.

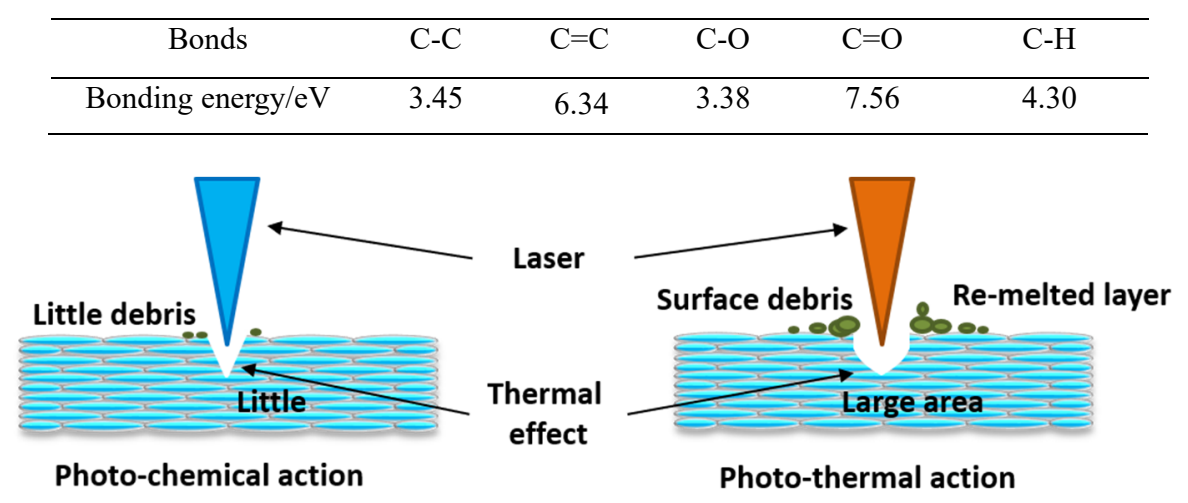

Fig. 4. Interaction of laser and PEEK surface. 
When the laser energy density increases to $Q=180$ $\mathrm{J} \cdot \mathrm{cm}^{-2}$, the interaction between laser photons and PEEK shows modification effect with significant multiphoton absorption. The melting degree of the interface region in the laser interaction region on PEEK surface is deepened. As the organic polymer melts at instantaneous high temperature, there are also a lot of gasification effect and $\mathrm{CO}_{2}$ and other gases produced by the breaking of PEEK intramolecular chemical bonds under pulse laser photochemical reaction. This kind of gas and gaseous substance expand rapidly and escape from the melt due to the effect of internal and external pressure difference, and then the melt cools rapidly [23, 24]. This effect makes the interface of laser-induced modification of PEEK substrate in the process of melting, gasification and solidification to form a dispersed flocculent microstructure, meanwhile, there are micro-holes in the microstructure, thus showing a special surface effect.

\subsection{Preparation of metal layer on the PEEK after laser modification}

Although PEEK material has excellent properties, its high insulation properties limit its application in radar, antenna and other related fields. It is necessary to carry out high conductivity and dense metallization on its surface to meet the relevant electrical properties of products. Therefore, the metallization of PEEK after laser induced treatment is analyzed in this paper.

In order to realize the following engineering application (conduction, welding and other practical needs), the electroless nickel coating which is commonly used in engineering is selected for metallization to carry out the coating test, and the influence of laser treatment with different energy density on the bonding strength of metal layer on the surface of PEEK is studied.

It is found that the original PEEK has a certain hydrophobicity, and the continuous water film failed to be formed after the oil is removed completely, which is not conducive to the deposition of metal layer on the surface.

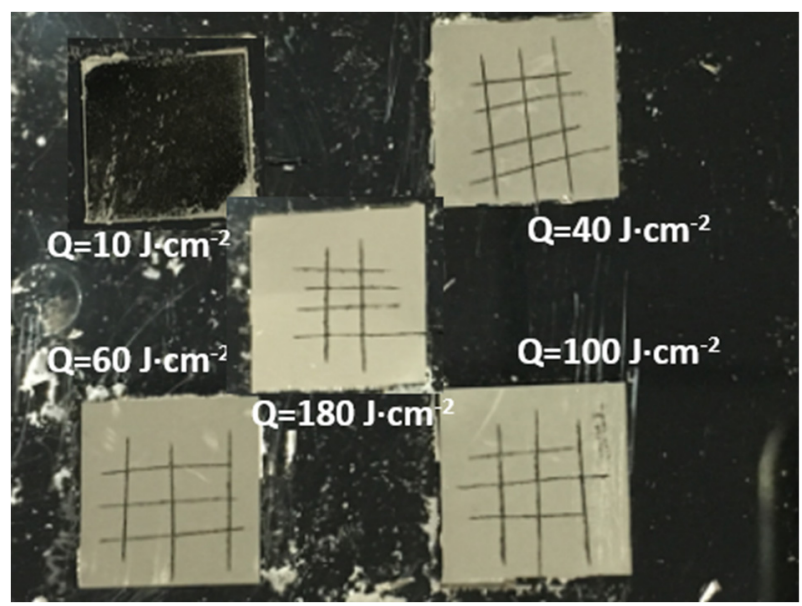

Fig. 5. Images of Ni-P alloys on the PEEK surface after laser treatments

The electroless nickel plating on the surface of PEEK substrate after different pulse laser energy density treatment presents different bonding strengths. The bonding strength of the surface coating measured by the grid method is shown in Fig. 5. It can be seen that when the energy density of pulse laser is only $10 \mathrm{~J} \cdot \mathrm{cm}^{-2}$, there is no bonding strength between the electroless nickel coating on the substrate, and the coating appears obvious peeling phenomenon. This may be due to the fact that although the micro gully three-dimensional morphology is formed in some areas of the PEEK surface after the pulsed laser-induced modification, the substrate still has obvious smooth micro-surface, which greatly reduces the friction between the metal layer and the substrate. The bonding strength of the metal layer on the substrate improved for the samples with the increased laser energy density. The microscopic metallographic photos of the coating shown in Fig. 6 also show that the electroless nickel coating on the PEEK is uniform, meanwhile, the grain is fine and dense. Therein, the corresponding chimeric structure is formed on the three-dimensional micro nano structure surface at the interface between electroless nickel coating and the PEEK with laser treatment. The coating crystal can completely penetrate into the micro uneven area of PEEK substrate, and connect into a dense structure without obvious defects, thus forming a highly dense and highly bonded metallized layer. 


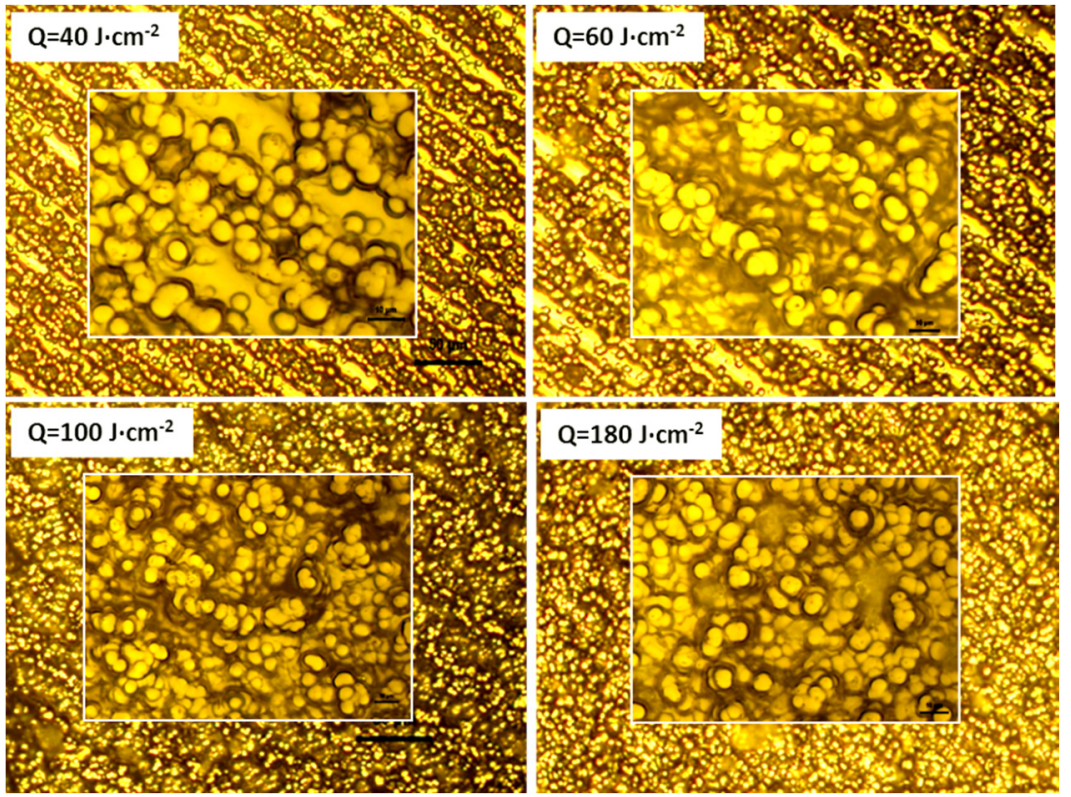

Fig. 6. Images of Ni-P alloys on the PEEK surface after laser treatments.

The electroless nickel coating on the PEEK after graduation is shown in Fig. 7. The results of graduation test show that when the laser energy density is $40 \mathrm{~J} \cdot \mathrm{cm}^{-2}$ and $180 \mathrm{~J} \cdot \mathrm{cm}^{-2}$, the bonding strength of the coating is

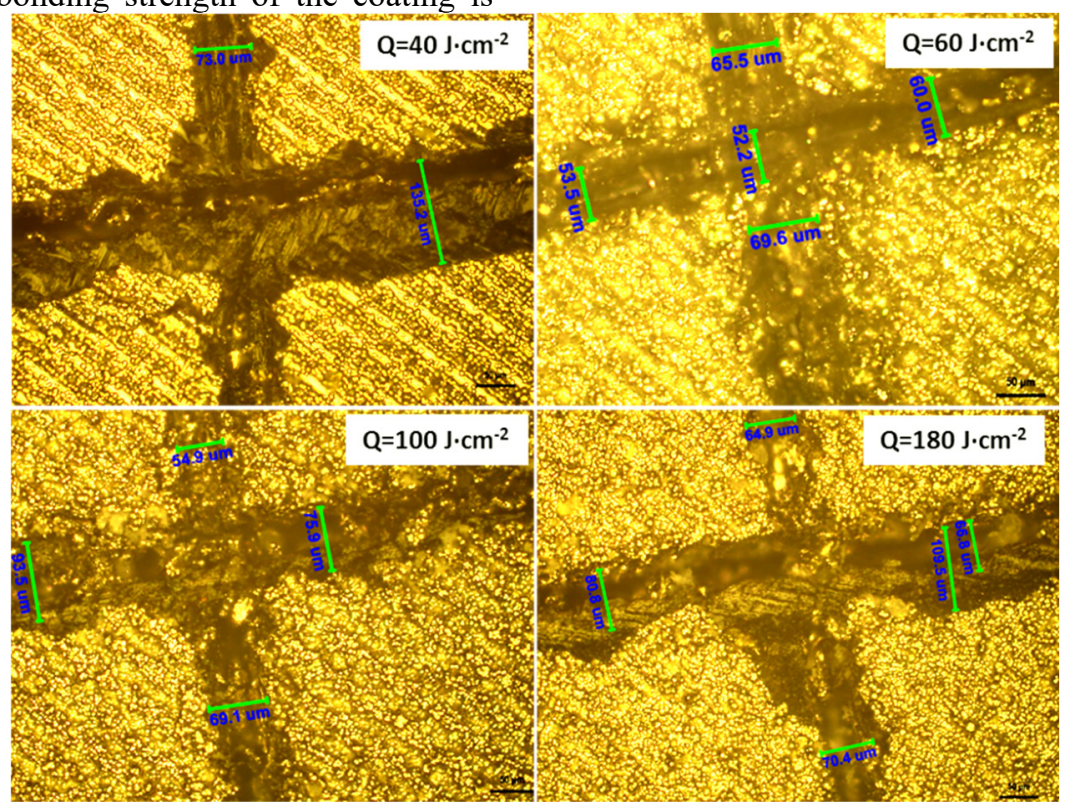

Fig. 7. Images of Ni-P alloys on the PEEK surface with laser treatments after scratch tests.

In order to meet the requirements of high and low temperature bonding strength control in the external environment, the bonding strength between the PEEK with laser-induced modification and electroless nickel coating has been further verified by high and low temperature thermal cycling test under atmospheric pressure. According to GJB $150.5 \mathrm{~A}-2009$, there is no bubbling, peeling or delamination in the coating after 10 cycles at $-196{ }^{\circ} \mathrm{C} \sim 100{ }^{\circ} \mathrm{C}$. It shows that there is a good bonding strength between the laser modified PEEK and the surface nickel layer. slightly lower than that of $\mathrm{Q}=60 \mathrm{~J} \cdot \mathrm{cm}^{-2}$ and $\mathrm{Q}=100$ $\mathrm{J} \cdot \mathrm{cm}^{-2}$, however, it can basically meet the detection requirements of grid method at the same.

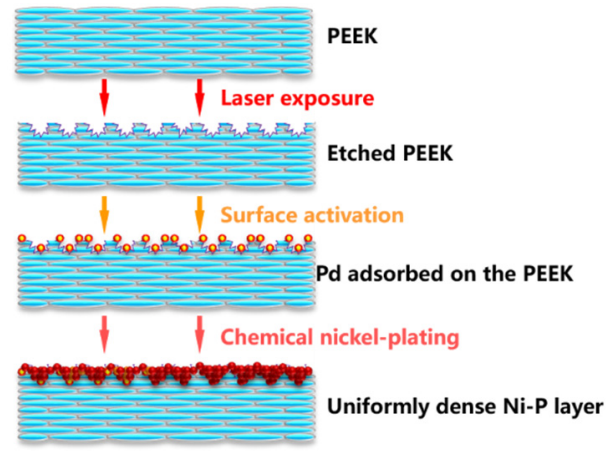

Fig. 8. Images of the process of Ni-P alloys deposition on the PEEK surface after laser treatments. 
Combined with the morphology characteristics of PEEK surface treated by pulsed laser with different energy density and the bonding strength difference of metal layer on the surface of PEEK, the following analysis is considered. When the laser energy density is lower $\left(\mathrm{Q}=10 \mathrm{~J} \cdot \mathrm{cm}^{-2}\right)$, the results show that the microstructure of the micro-surface of PEEK is obviously different from that of the smooth microstructure boundary area, which leads to the failure to form an effective high adhesion point between the metal particles and the substrate when they are deposited in the smooth non etched surface. When the chemical nickel coating is grown in a two-dimensional manner, the three-dimensional adhesion between the metal particles and the highly chemically inert PEEK substrate would fail to realize the physical chimeric effect of the micro-structure, so as to the electroless nickel coating without binding force. As a result, and the subsequent bonding strength test shows the peeling and peeling phenomenon of the metal layer.

However, the uniformity of the etched surface of the PEEK with the laser treatment at $\mathrm{Q}=40 \mathrm{~J} \cdot \mathrm{cm}^{-2}$ is not enough, resulting in the formation of a more obvious undulating structure. Thus, the subsequent electroless nickel coating deposition is uniform, but the bonding strength of the coating would reach the optimal state in failure.

When the laser energy density increased to $Q=60$ $\mathrm{J} \cdot \mathrm{cm}^{-2}$ and $\mathrm{Q}=100 \mathrm{~J} \cdot \mathrm{cm}^{-2}$, the surface of PEEK after laser-induced modification shows obvious photothermal effect, accompanied by weak photochemical effect, resulting in superior etching uniformity. The surface of PEEK is uniformly covered with molten or solidified products, and has a small amount of void structure formed by vaporization cooling, which effectively improves the bonding strength between the surface coating and PEEK.

Nevertheless, when the laser energy density further increases to $\mathrm{Q}=180 \mathrm{~J} \cdot \mathrm{cm}^{-2}$, In the case of etching effect, more dispersed floccules are produced on the surface of PEEK. The structure of the dispersed floccules is loose and in low density. In consequence, there is no good bonding strength between the coating and the substrate. Depositing a metal layer on the surface of the dispersed floccules can not improve the bonding strength between the coating and the substrate, but reduce the bonding strength between them, leading to the peeling of the coating.

\section{Conclusions}

In this paper, the surface of PEEK was modified by 1064 $\mathrm{nm}$ pulse infrared laser, and the effect of laser treatment with different energy density on the surface morphology of PEEK was studied here. The results show that when the laser energy density is low $\left(\mathrm{Q}<60 \mathrm{~J} \cdot \mathrm{cm}^{-2}\right)$, the surface of PEEK is periodically undulating groove structure with a small number of holes. As the laser energy density is high $\left(\mathrm{Q} \geq 60 \mathrm{~J} \cdot \mathrm{cm}^{-2}\right)$, the surface of PEEK is uniformly covered with a layer of melt or re-solidified product after melting. The analyses shows that the main reason is that the interaction mechanism between laser photons and the surface molecules of PEEK is different during laser treatment. When the laser energy density is low, there is mainly photothermal effect, and the thermal effect accumulation makes the surface thermal expansion, melting and vaporization. However, when the laser energy density is high, the photothermal effect on the surface is enhanced, and has some photochemical effect. Therein, the chemical bond breaks during photo-pyrolysis, and the amount of PEEK on the surface increases greatly after melting or re-solidification, then gradually connects into sheets and accumulates.

Electroless nickel plating has been carried out on the surface of PEEK modified by laser etching. The electroless nickel coating on the PEEK is peeled off at the low laser energy density $\left(\mathrm{Q}=10 \mathrm{~J} \cdot \mathrm{cm}^{-2}\right)$. The nickel coating is compact and uniform as the laser energy density is increased $\left(\mathrm{Q}>10 \mathrm{~J} \cdot \mathrm{cm}^{-2}\right)$. The lattice test shows that the laser-induced metal layer on PEEK has good adhesion. In this study, the metal layer on the surface of PEEK has been prepared under laser-induced effect, which provides technical support for the application of PEEK in radar antenna and other aerospace fields.

\section{References}

1. S. Verma, N. Sharma, S. Kango, S. Sharma, Eur. Polym. J. 147, 110295 (2021).

2. H. Ma, A. Suonan, J. Zhou, Q. Yuan, L. Liu, X. Zhao, X. Lou, C. Yang, D. Li, Y. Zhang, Arab. J. Chem., 14, 102977 (2021).

3. Y. Niu, S. Zheng, P. Song, X. Zhang, C. Wang, Compos. B. Eng. 108715 (2021).

4. L. Pan, F. Wang, X. Pang, J Mater Sci. 54, 14728 (2019).

5. T. Wan, L. Li, M. Guo. J. Mater. Sci., 54, 11179 (2019).

6. E.I. González-Castillo, T. Costantini, M.S.P. Shaffer, J. Mater. Sci. 55, 8881 (2020).

7. L. Mathur, In-Ho Kim, A. Bhardwaj, B. Singh, J. Park, S. Song, Compos. B. Eng., 202, 108405 (2020).

8. J. Wang, Y. Sun, W. Bi, Z. Jiang, M. Zhang, J. Membr. Sci. 616, 118418 (2020).

9. F. Sargın, G. Erdoğan, K. Kanbur, A. Türk, Surf. Coat. Technol. 2021, 126965.

10. P. Xian, Y. Chen, S. Gao, J. Qian, W. Zhang, A. Udduttula, N. Huang, G. Wan, Surf. Coat. Technol. 401, 126282 (2020).

11. M. Thiruchitrambalam, D. Bubesh Kumar, D. Shanmugam, M. Jawaid, Mater. Today. 33, 1085 (2020).

12. X.L. Zhou, A.F. Chen, J.C. Liu, X.K. Wu, J.S. Zhang, Surf. Coat. Technol. 206, 132 (2011).

13. X. Chen, Y. Su, D. Reay, S. Riffat, Renew. Sust. Energ. Rev. 60, 1367 (2016). 
14. C. Chen, X. Xie, Y. Xie, X. Yan, C. Huang, S. Deng, Z. Ren, H. Liao, Surf. Coatings Technol. 342, 209 (2018).

15. Y. Wang, W. Müller, A. Rumjahn, F. Schmidt, A. Schwitalla, J. Mech Behav Biomed Mater. 115, 104250 (2021).

16. L. Yang, Y. Ohki, N. Hirai, S. Hanada, Polym. Degrad. Stab. 142, 117-128 (2017).

17. T Zhai, L Z Di, D Yang. ACS Appl. Mater. Interfaces 5, 12499 (2013).

18. Z K Wang, H Y Zheng, Y C Lam. Applied Surface Science (S0164-4332), 257(24):10427 (2011).

19. H. Pazokian, S. Jelvani, M. Mollabashi, J. Barzin, G. Azizabadi Farahani, Appl. Surf. Sci. 257, 6186 (2011).

20. A. Ramazani S. A., S. Mousavi, E. Seyedjafari, R. Poursalehi, S. Sareh, K. Silakhori, A. Akbar Poorfatollah, A. Shamkhali, Mater. Sci. Eng. C 29, 1491 (2009).

21. W. Zhang, Z. Chen, J. Chromatogr. A. 1278, 29 (2013).

22. Riveiro A, Soto R, Comesana R, et al. Applied Surface Science(S0164-4332), 258(23):9437 (2012).

23. A. Hartwig, J. Hunnekuhl, G. Vitr, S. Dieckhoff, F. Vohwinkel, O.D. Hennemann, J. Appl. Polym. Sci. 64, 1091 (1997).

24. P Laurens, M Ould Bouali, F Meducin, B Sadras, Appl. Surf. Sci. 154, 211-216 (2000). 